\title{
Instability of sliding Luttinger liquid
}

\author{
V Fleurov ${ }^{1}$, V Kagalovsky ${ }^{2,5}$, I V Lerner ${ }^{3}$ and I V Yurkevich Ka $^{4,5}$ \\ ${ }^{1}$ Raymond and Beverly Sackler Faculty of Exact Sciences, School of Physics and \\ Astronomy, Tel-Aviv University - Tel-Aviv 69978, Israel \\ ${ }^{2}$ Shamoon College of Engineering - Beer-Sheva 84105, Israel \\ ${ }^{3}$ University of Birmingham, School of Physics \& Astronomy - Birmingham B15 2TT, \\ UK \\ ${ }^{4}$ Aston University, School of Engineering \& Applied Science - Birmingham B4 7ET, \\ UK \\ ${ }^{5}$ Institute of Fundamental and Frontier Sciences, University of Electronic Science \\ and Technology of China - Chengdu 610054, People's Republic of China \\ E-mail: victork@sce.ac.il
}

August 2017

\begin{abstract}
We revise a phase diagram for the sliding Luttinger liquid (SLL) of coupled one-dimensional quantum wires packed in two- or three-dimensional arrays in the absence of a magnetic field. We analyse whether physically justifiable (reasonable) inter-wire interactions, i.e. either the screened Coulomb or "Coulomb-blockade" type interactions, stabilise the SLL phase. Calculating the scaling dimensions of the most relevant perturbations (the inter-wire single-particle hybridisation, charge-density wave, and superconducting inter-wire couplings), we find that their combination always destroys the SLL phase for the repulsive intra-wire interaction. However, suppressing the inter-wire tunnelling of repulsive fermions (when the charge-density wave is the only remaining perturbation), one can observe a stability region emerging due to the inter-wire forward scattering interaction.
\end{abstract}

Keywords: Luttinger liquid

Submitted to: J. Phys.: Condens. Matter

\section{Introduction}

The Luttinger liquid (LL) describes one-dimensional interacting systems with a linear (or linearised) spectrum $[1,2,3]$. The interaction strongly enhances the impact of impurities leading to a zero-temperature metal-insulator transition in the presence of either disorder [4] or even a single impurity [5,6], both being described by the renormalisation group $(\mathrm{RG})$ approach. Progress in the fabrication of low-dimensional nanostructures based 
on carbon nanotubes $[7,8,9,10]$, semiconductor and metallic nanowires [11, 12], selfassembled DNA scaffolds $[13,14]$, etc., revived the interest to theoretical studies of the LL superstructures. Theoretically, the most challenging bunch of problems in this field is the crossover from the 1D LL behaviour of an individual metallic wire to a $2 \mathrm{D}$ or $3 \mathrm{D}$ Fermi-liquid (FL) behaviour of ensembles of coupled 1D wires. Such finite systems may support various topological states with gapless modes propagating through the edge wires [15].

The conductivity of multi-channel (quasi-one-dimensional) strongly-correlated systems demonstrates power law temperature dependence. It has been experimentally observed in many quasi-1D systems, including carbon nano-tubes [16], semiconductor nanowires [17], MoSe nanowires [18], $\mathrm{NbSe}_{3}$ nanowires [19], gold nanowires [20], polymer fibers [21] and newly discovered $\mathrm{NB}_{2} \mathrm{PdS}_{5}$ nanowires [22]. These observations can be explained within multi-channel Luttinger liquid model that neglects singleparticle backscattering and many-particle scattering processes. The single-particle backscattering results from disorder and can be neglected in a clean (ballistic) translation invariant wires whereas many-particle scatterings are inherent in strongly correlated systems. Many-particle processes are typically weaker but they are renormalised by interaction and may become dominant at low temperatures. The fact that they are not observed in some of the experiments cited above can be explained by relatively high temperatures at which the multi-particle processes are still ineffective. Hightemperature phase of multi-channel strongly correlated fermionic systems, therefore, are well described by the so-called sliding Luttinger liquid (SLL) [25, 26, 27, 28, 29, 31]. Nevertheless, at lower temperatures quasi-1D systems fall into either a superconducting (SC) [22] or charge density wave (CDW) [19] states. It is also known that the inter-wire electron tunnelling is a relevant perturbation which results in the transition from the LL to Fermi liquid (FL) phase [23, 24]. The aim of this manuscript is to establish whether the LL fixed point remains stable in a phase of the sliding Luttinger liquid assuming realistic intra- and inter-wire interactions.

In the sLL phase, the canonic phase - density variables $\left(\varphi_{j}, \theta_{j}\right)$ describing bosonised degrees of freedom in each wire $j$ are invariant under the constant shifts. The phase remains stable as long as three distinct inter-wire processes are all RG irrelevant. Namely, these processes are the single-particle (SP) and the particle-hole inter-wire tunnelling that may result in a transverse charge density wave (CDW), and the twoparticle hopping resulting in a superconducting (SC) state. The SP processes become irrelevant when a spin gap appears due to an attraction between opposite spins (the Luther-Emery regime $[24,32,33])$. Then the stability of the SLL fixed point would be ensured if the remaining SC and CDW interactions also become irrelevant [28, 29]. In the spin-polarised regime, the system becomes effectively spinless, but the SP processes are not necessarily irrelevant. Then all three types of interaction, CDW, SC, and SP, must be simultaneously irrelevant in order to ensure the stability of SLL. It requires a fine tuning of model parameters to achieve a stability against all three perturbations. Even assuming unrealistic interaction between wires [31, 30] (in attempt to save SLL 
model) the stability region of parameters turns into a narrow strip along the boundary beyond which the Wentzel-Bardeen [37] instability completely breaks the Luttinger liquid approach. The special shape of the transverse wave vector dependence of the Luttinger parameter is used in [31,30], whose microscopic derivation is not obvious. Moreover, it is quite clear that the result is extremely sensitive to the choice of the shape of this dependence and the values of the parameters. In what follows, we do not assume that the inter-wire interaction is different to a standard (screened) Coulomb interaction, and his fact leads to a conclusion drastically different from that in [31].

In this letter we study a sliding Luttinger liquid phase in a system of coupled parallel quantum wires packed in two- and three-dimensional arrays.

\section{The model}

After the standard bosonisation [24], the density fluctuations and current in the $i^{\text {th }}$ wire are parameterised in terms of two bosonic fields $\theta_{i}$ and $\varphi_{i}$ as $\delta \rho_{i}=\frac{1}{\pi} \partial_{x} \theta_{i}$, and $j_{i}=\frac{1}{\pi} \partial_{x} \varphi_{i}$. We assume spin-gapped situation when only charge degrees of freedom should be kept. Introducing the vector notations for the two fields describing a set of $N$ wires,

$$
\begin{aligned}
& \boldsymbol{\theta}=\left\{\theta_{1}, \ldots, \theta_{N}\right\}, \\
& \boldsymbol{\varphi}=\left\{\varphi_{1}, \ldots, \varphi_{N}\right\},
\end{aligned}
$$

one writes the Lagrangian density of the set as

$$
\mathcal{L}=\frac{1}{8 \pi}\left[2 \partial_{t} \boldsymbol{\varphi}^{\mathrm{T}} \partial_{x} \boldsymbol{\theta}-\partial_{x} \boldsymbol{\theta}^{\mathrm{T}} \mathrm{V}_{\theta} \partial_{x} \boldsymbol{\theta}-\partial_{x} \boldsymbol{\varphi}^{\mathrm{T}} \mathrm{V}_{\varphi} \partial_{x} \boldsymbol{\varphi}\right]
$$

Here the matrices $\mathrm{V}_{\theta}$ and $\mathrm{V}_{\varphi}$ are diagonal in the absence of inter-wire interactions, with the elements expressed in terms of the velocity $v_{i}$ and the Luttinger parameter $K_{i}$ in

each wire as $V_{\theta}^{i j}=\delta_{i j}\left(v_{i} / K_{i}\right)$ and $V_{\varphi}^{i j}=\delta_{i j} v_{i} K_{i}$. Adding the inter-wire interactions makes these matrices non-diagonal,

$$
\begin{aligned}
V_{\theta}^{i j} & =\delta_{i j}\left(v_{i} / K_{i}\right)+U_{\theta}^{i j}, \\
V_{\varphi}^{i j} & =\delta_{i j} v_{i} K_{i}+U_{\varphi}^{i j},
\end{aligned}
$$

where the off-diagonal matrix elements $U_{\theta}^{i j}$ and $U_{\varphi}^{i j}$ describe the density-density and current-current interaction strengths between the $i^{\text {th }}$ and $j^{\text {th }}$ wires. In the presence of the inter-wire interaction, the local field correlators with Lagrangian density Eq. (3) can be written in matrix form as

$$
\begin{aligned}
& \left\langle\boldsymbol{\theta}(t) \otimes \boldsymbol{\theta}^{\mathrm{T}}\left(t^{\prime}\right)\right\rangle=-2 \mathrm{~K} \ln \left(t-t^{\prime}\right), \\
& \left\langle\boldsymbol{\varphi}(t) \otimes \boldsymbol{\varphi}^{\mathrm{T}}\left(t^{\prime}\right)\right\rangle=-2 \mathrm{~K}^{-1} \ln \left(t-t^{\prime}\right),
\end{aligned}
$$

where the Luttinger matrix $\mathrm{K}$ is defined $[34,35,36]$ by the matrix equation

$$
\mathrm{KV}_{\theta} \mathrm{K}=\mathrm{V}_{\varphi}
$$

which always has a unique solution for real symmetric and positive definite matrices $V_{\theta}$ and $V_{\varphi}$. The name is justified by the fact that, in the absence of inter-wire interactions, $\mathrm{K}=\operatorname{diag}\left\{\mathrm{K}_{\mathrm{i}}\right\}$, and Eqs. 6 are reduced to the standard single-wire expressions. 


\section{Perturbations}

It is convenient to write operators corresponding to the three inter-wire processes defined in the introduction (CDW originating from Coulomb interaction, and SP/SC tunneling processes) in terms of the creation and annihilation operators for the left-moving $\left(\hat{L}_{i}^{\dagger}\right.$ and $\left.\hat{L}_{i}\right)$ and right-moving $\left(\hat{R}_{i}^{\dagger}\right.$ and $\left.\hat{R}_{i}\right)$ particles in the $i^{\text {th }}$ wire, with $\hat{L}_{i} \sim \mathrm{e}^{i \theta_{i}^{L}}$ and $\hat{R}_{i} \sim \mathrm{e}^{i \theta_{i}^{R}}$, where the bosonic variables for the left- and right-movers are $\theta_{i}^{L, R} \equiv \varphi_{i} \pm \theta_{i}$. Then the matrix elements of the three potentially relevant inter-wire couplings are given by

$$
\begin{aligned}
& \hat{L}_{i j}^{\mathrm{cdw}} \sim \hat{R}_{i}^{\dagger} \hat{L}_{i} \hat{L}_{j}^{\dagger} \hat{R}_{j} \sim \cos \left[\theta_{i}-\theta_{j}\right], \\
& \hat{L}_{i j}^{\mathrm{sc}} \sim \hat{R}_{i}^{\dagger} \hat{L}_{i}^{\dagger} \hat{L}_{j} \hat{R}_{j} \sim \cos \left[\varphi_{i}-\varphi_{j}\right], \\
& \hat{L}_{i j}^{\mathrm{sp}} \sim \hat{R}_{i}^{\dagger} \hat{R}_{j} \sim \mathrm{e}^{i\left(\theta_{i}-\theta_{j}\right) / 2} \mathrm{e}^{i\left(\varphi_{i}-\varphi_{j}\right) / 2}, .
\end{aligned}
$$

The corresponding scaling dimensions are straightforward to derive using field correlators Eq. (6); the results are expressed in terms of matrix elements of the Luttinger matrix $\mathrm{K}$ and its inverse $\mathrm{K}^{-1}$ :

$$
\begin{aligned}
& \Delta_{i j}^{\mathrm{cdw}} \equiv \operatorname{dim}\left[\hat{L}_{i j}^{\mathrm{cdw}}\right]=K_{i i}+K_{j j}-2 K_{i j}, \\
& \Delta_{i j}^{\mathrm{sc}} \equiv \operatorname{dim}\left[\hat{L}_{i j}^{\mathrm{sc}}\right]=\left(\mathrm{K}^{-1}\right)_{i i}+\left(\mathrm{K}^{-1}\right)_{j j}-2\left(\mathrm{~K}^{-1}\right)_{i j}, \\
& \Delta_{i j}^{\mathrm{sp}} \equiv \operatorname{dim}\left[\hat{L}_{i j}^{\mathrm{sp}}\right]=\frac{1}{4}\left[\Delta_{i j}^{\mathrm{cdw}}+\Delta_{i j}^{\mathrm{sc}}\right] .
\end{aligned}
$$

The stability condition (i.e. the irrelevance of all the three perturbations) for a onedimensional system is that all the three scaling dimensions are greater than the physical dimension, $1+1=2$ :

$$
\begin{aligned}
& \Delta_{i j}^{\mathrm{cdw}} \geq 2, \Delta_{i j}^{\mathrm{sc}} \geq 2, \\
& \Delta_{i j}^{\mathrm{cdw}}+\Delta_{i j}^{\mathrm{sc}} \geq 8 .
\end{aligned}
$$

The last inequality is potentially most stringent so that the single-particle hybridisation might be dangerous for the stability of the SLL phase even when both CDW and SC processes are irrelevant. Let us stress that, as usual, the most relevant process does not necessarily makes the strongest impact on observables. The impact also depends on bare values of the inter-wire couplings omitted in Eqs. $(9,10,11)$. Since both $\hat{L}_{i j}^{\mathrm{sp}}$ and $\hat{L}_{i j}^{\text {sc }}$ involve tunnelling between the $i^{\text {th }}$ and $j^{\text {th }}$ wires, their bare values can be much smaller than that of $\hat{L}_{i j}^{\mathrm{cdw}}$ that involves only virtual processes. However, the bare values are totally irrelevant at $T=0$ so that the results based on the analysis of the $\mathrm{RG}$ dimensions should survive at low enough temperatures. In the last section, we will come back to the situation when a direct inter-wire tunnelling is suppressed so that only CDW processes should be taken into account.

\section{Identical wires}

If all the wires are identical and packed into a 2D or 3D array, the labels $i$ are replaced by lattice vectors $\boldsymbol{R}$ where $\boldsymbol{R} \subset \mathcal{L}$ and $\mathcal{L}$ is the one- or two-dimensional lattice of wires. 
Assuming the interactions to be translationally invariant $\left(V_{i j} \rightarrow V_{\mid \boldsymbol{R}-\boldsymbol{R}^{\prime}} \mid\right)$, Eq. (8) for the Luttinger matrix takes the following form:

$$
\sum_{\boldsymbol{R}_{1}, \boldsymbol{R}_{2} \subset \mathcal{L}} K_{\boldsymbol{R}-\boldsymbol{R}_{1}} V_{\boldsymbol{R}_{1}-\boldsymbol{R}_{2}}^{\theta} K_{\boldsymbol{R}_{2}-\boldsymbol{R}^{\prime}}=V_{\boldsymbol{R}-\boldsymbol{R}^{\prime}}^{\varphi},
$$

where $\boldsymbol{r} \equiv \boldsymbol{R}-\boldsymbol{R}^{\prime} \subset \mathcal{L}$ and lengths are measured in units where the inter-wire distance is put to 1 . This equation is solved via the Fourier transform:

$$
\begin{aligned}
& K_{\boldsymbol{r}}=\int \frac{\mathrm{d}^{d} q}{(2 \pi)^{d}} K_{\boldsymbol{q}} \mathrm{e}^{i \boldsymbol{q} \boldsymbol{r}}, \quad K_{\boldsymbol{q}}=\sqrt{\frac{V_{\boldsymbol{q}}^{\varphi}}{V_{\boldsymbol{q}}^{\theta}}}, \\
& V_{\boldsymbol{q}}^{\theta / \varphi}=V_{0}^{\theta / \varphi}+\sum_{\boldsymbol{r} \neq \mathbf{0}} U_{\boldsymbol{r}}^{\theta / \varphi} \mathrm{e}^{-i \boldsymbol{q} \boldsymbol{r}} .
\end{aligned}
$$

Here and below the momentum integration is carried out over a Brillouin zone. This results in the following expressions for the scaling dimensions for the coupling between wires separated by the lattice vector $\boldsymbol{r}$ :

$$
\begin{aligned}
& \Delta_{r}^{\mathrm{cdw}}=2 \int \frac{\mathrm{d}^{d} q}{(2 \pi)^{d}} K_{\boldsymbol{q}}[1-\cos \boldsymbol{q} \boldsymbol{r}] \\
& \Delta_{r}^{\mathrm{sc}}=2 \int \frac{\mathrm{d}^{d} q}{(2 \pi)^{d}} K_{\boldsymbol{q}}^{-1}[1-\cos \boldsymbol{q} \boldsymbol{r}], \\
& \Delta_{r}^{\mathrm{sp}}=\frac{1}{4}\left[\Delta_{r}^{\mathrm{cdw}}+\Delta_{\boldsymbol{r}}^{\mathrm{sc}}\right] .
\end{aligned}
$$

We have assumed above that the wires are arranged into a simple Bravais lattice. For a non-Bravais lattice, the summation in matrix equation (Eq. (17)) should be carried out over all the sites in an elementary cell, with the appropriate changes to the Fouriertransform solution.

\section{Interactions}

In the absence of inter-wire interactions, when $\mathrm{U}=0$ in Eq. (4), the Luttinger matrix is diagonal, with all elements equal to $K$. Assuming that only the nearest neighbouring wires are coupled for all the three perturbations, the scaling dimensions are reduced to

$$
\Delta_{0}^{\mathrm{cdw}}=2 K, \quad \Delta_{0}^{\mathrm{sC}}=\frac{2}{K}, \quad \Delta_{0}^{\mathrm{sp}}=\frac{1}{2}\left[K+\frac{1}{K}\right] .
$$

Obviously, there is no value of $K$ for which all the scaling dimensions are above 2 so that the SLL is unstable, at least in the absence of long-range interactions. A weak short-range inter-wire interaction cannot stabilise the SLL phase since it gives only small corrections to the RG dimensions in Eq. (23) which are never simultaneously close to 2. Let us consider the case of a weakly screened Coulomb interaction, with $U_{\boldsymbol{r}}^{\theta / \varphi}=\alpha_{\theta / \varphi} e^{-\kappa r} / r$ in Eq. (18) and $\kappa \ll 1$. Then, we represent $V_{\boldsymbol{q}}^{\theta / \varphi}$ in Eq. 18 for $2 D$ and $3 D$ arrays as follows:

$$
\begin{aligned}
V_{\boldsymbol{q}}^{\theta / \varphi} & =V_{0}^{\theta / \varphi}\left[1+\alpha_{\varphi / \theta} u_{\boldsymbol{q}}\right], \\
u_{\boldsymbol{q}}^{(2 D)} & =-\ln \left[1-2 \beta \cos q+\beta^{2}\right], \quad \beta \equiv \mathrm{e}^{-\kappa},
\end{aligned}
$$



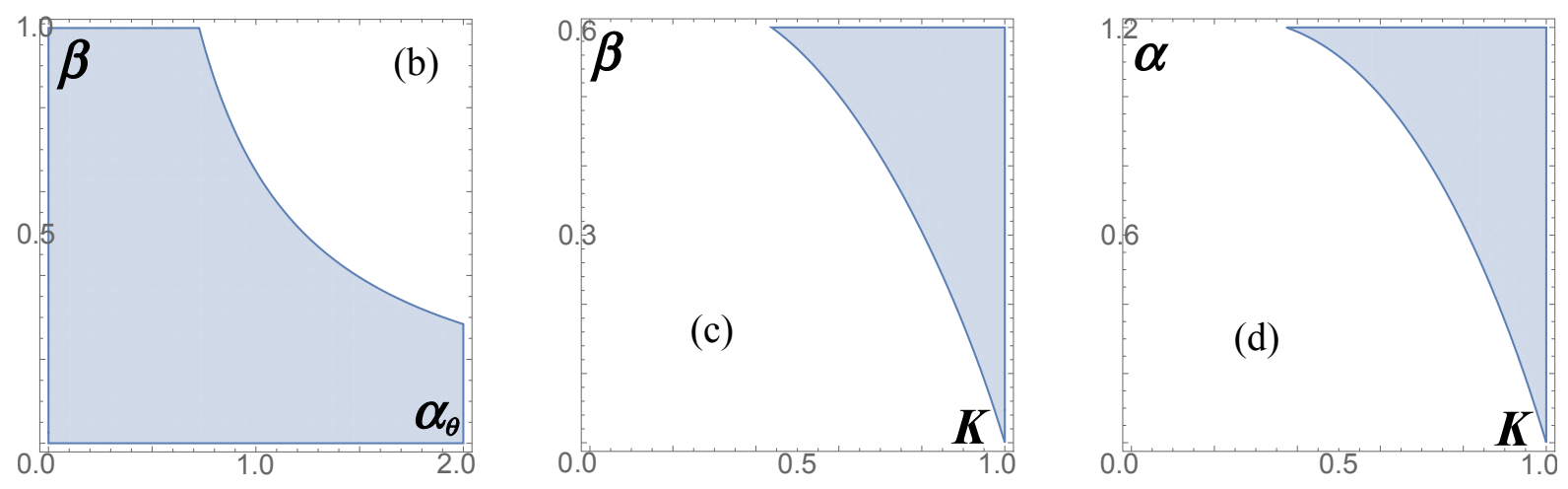

Figure 1. Stability diagram of the SLL phase for a fermionic system with the intrawire repulsion $(K \leqslant 1)$ without a direct inter-wire tunnelling. Here the strength of the density-density inter-wire interaction is parametrised by $\alpha_{\theta}$, and its range by the screening parameter $\beta \equiv \mathrm{e}^{-\kappa}$ (with $\kappa^{-1}$ being the screening length measured in units with the inter-wire distance equal 1): (a) in $\alpha_{\theta}-\beta$ plane for a system without the intra-wire interaction $(K=1)$; (b) in $K-\beta$ plane for a system with $\alpha_{\theta}=1$; (c) in $K-\alpha_{\theta}$ plane for a system with $\beta=0.5$. Coloured (light blue) parts in Figs. 1b-d show the stability regions, whereas white parts correspond to the regions of sLL instability.

$$
u_{\boldsymbol{q}}^{(3 D)}=\sum_{\boldsymbol{n} \neq 0} \frac{\exp \left[-\kappa \sqrt{n_{1}^{2}+n_{2}^{2}}\right]}{\sqrt{n_{1}^{2}+n_{2}^{2}}} \mathrm{e}^{-i\left(q_{1} n_{1}+q_{2} n_{2}\right)} .
$$

The Fourier-transform of Luttinger matrix K, Eq. 18, is expressed via $u_{\boldsymbol{q}}$ as

$$
\begin{aligned}
& K_{\boldsymbol{q}} \equiv K Q(\boldsymbol{q}) \\
& Q(\boldsymbol{q})=\sqrt{\frac{1+\alpha_{\varphi} u_{\boldsymbol{q}}}{1+\alpha_{\theta} u_{\boldsymbol{q}}}} .
\end{aligned}
$$

The expression under the square root could become negative for some $q$ if any of the inter-wire interaction strength, $\alpha_{\varphi}$ or $\alpha_{\theta}$, exceeds $\alpha_{\mathrm{WB}} \equiv(2 \ln (1+\beta))^{-1}$, the boundary of the Wentzel-Bardeen [37] instability, typical for any multi-channel system. The standard LL approach is not valid there so that we assume that both interaction strength are bounded from above. The scaling dimensions for the next-neighbours coupling are then expressed as

$$
\Delta^{\mathrm{cdw}}=2 K\left\langle Q_{q}\right\rangle, \quad \Delta^{\mathrm{sc}}=2 K^{-1}\left\langle Q_{q}^{-1}\right\rangle
$$

where the angular brackets are defined by

$$
\langle f\rangle \equiv\left\{\begin{array}{c}
\int_{0}^{2 \pi} \frac{\mathrm{d} q}{2 \pi} f_{q}(1-\cos q), \quad 2 D \\
\int_{0}^{2 \pi} \frac{\mathrm{d} q_{1}}{2 \pi} \frac{\mathrm{d} q_{2}}{2 \pi} f_{\boldsymbol{q}}\left(1-\cos q_{1}\right), \quad 3 D .
\end{array}\right.
$$

The inter-wire interaction opens up a potential region of stability as, in contrast to the case of isolated channels, the inequalities $\Delta^{\mathrm{cdw}}>2$ and $\Delta^{\mathrm{sc}}>2$ can be 
both satisfied provided that $\left\langle Q_{q}\right\rangle^{-1}<K<\left\langle Q_{q}^{-1}\right\rangle$. The third condition of stability, $\Delta^{\mathrm{sp}}=\frac{1}{4}\left(\Delta^{\mathrm{cdw}}+\Delta^{\mathrm{sc}}\right)>2$ is more stringent, as it can be satisfied simultaneously with the two previous ones only if

$$
\left\langle Q_{q}\right\rangle\left\langle Q_{q}^{-1}\right\rangle>3
$$

and either $\left\langle Q_{q}\right\rangle^{-1}<K<K_{+}$or $K_{-}<K<\left\langle Q_{q}^{-1}\right\rangle$, where $K_{ \pm}=$

$2\left\langle Q_{q}\right\rangle^{-1}\left[1 \pm\left(1-\frac{1}{4}\left\langle Q_{q}\right\rangle^{-1}\left\langle Q_{q}^{-1}\right\rangle\right)^{\frac{1}{2}}\right]$. For repulsive fermions, $K<1$, this can only happen when $\left\langle Q_{q}\right\rangle>1$. When we study numerically these stability conditions, we find that in for $2 \mathrm{D}$ and $3 \mathrm{D}$ packing there is no stability region of fermion SLL model.

\section{6. 'Coulomb-blockade' interaction}

Now we consider an ultimate long-range interaction, independent on the inter-wire distance. This model interaction can be used simply to test the extreme case of longrange interaction that allows analytic solution. On the other hand, such a model has a region of applicability in the problems related to interacting Kramers' doublets in a topological insulator where doublets form a set of 'channels' not separated spatially from each other. In this case the interaction matrices (in proper units) have the form:

$$
\mathrm{V}=(1-\alpha) \mathbf{1}+\alpha \mathrm{E} \Leftrightarrow V_{i j}=\delta_{i j}+\alpha\left[1-\delta_{i j}\right] .
$$

where all elements of matrix $\mathrm{E}$ are equal to unity. The coefficients $\alpha \rightarrow \alpha_{\theta, \varphi}$ for the two types of interaction in Eq. 4, All such matrices commute with each other. Thus, the solution of Eq. (8) for the Luttinger matrix is

$$
\mathrm{K}=\mathrm{V}_{\varphi}^{1 / 2} \mathrm{~V}_{\theta}^{-1 / 2}
$$

Assuming a finite number of wires $(=N)$ and noticing that $\mathrm{E}^{2}=N \mathrm{E}$, one expresses $\mathrm{V}^{-1}$ and $\mathrm{V}^{1 / 2}$ as

$$
\begin{aligned}
& \mathrm{V}^{-1}=\frac{1}{1-\alpha}\left[\mathbb{1}-\frac{\alpha}{N \alpha+1-\alpha} \mathrm{E}\right], \\
& \mathrm{V}^{\frac{1}{2}}=\sqrt{1-\alpha}\left[\mathbb{1}+\frac{1}{N}\left(\sqrt{1+\frac{N \alpha}{1-\alpha}}-1\right) \mathrm{E}\right] .
\end{aligned}
$$

Using this expressions one finds, with $c_{\varphi / \theta}=\frac{\alpha_{\varphi / \theta}}{1-\alpha_{\varphi / \theta}}$ :

$$
\mathrm{K}=K \sqrt{\frac{1-\alpha_{\varphi}}{1-\alpha_{\theta}}}\left[\mathbb{1}+\frac{1}{N}\left(\sqrt{\frac{N c_{\varphi}+1}{N c_{\theta}+1}}-1\right) \mathrm{E}\right] .
$$

Here again both interaction strength are bounded from above, $\alpha_{\theta, \varphi}<1$, by the WentzelBardeen [37] instability. The inverse matrix $\mathrm{K}^{-1}$ is obtained by swapping $\theta \leftrightarrow \varphi$. Substituting these expressions into Eqs. $(12,13,14)$, the off-diagonal elements cancel out, and only the diagonal ones contribute to the scaling dimensions:

$$
\Delta^{\mathrm{cdw}}=2 K Q, \quad \Delta^{\mathrm{sc}}=\frac{2}{K Q}, \quad Q=\sqrt{\frac{1-\alpha_{\varphi}}{1-\alpha_{\theta}}} .
$$


Thus, although the long-range (screened) Coulomb inter-wire interaction tends to stabilise the SLL phase, the infinite-range 'Coulomb-blockade' interaction simply renormalises the effective Luttinger parameter $(K \rightarrow Q K)$ and reproduces the results without inter-wire interactions, Eq. (23). Therefore, when all three perturbations, CDW, SC and SP , exist, there is no stable SLL-phase (where all the perturbations are irrelevant) for arrays of $1 \mathrm{D}$ channels and for all reasonable forms of intra- and interwire repulsions.

\section{Suppressed inter-channel scattering}

Here we show that a stable SLL can only be realised in a system without a direct interchannel tunnelling. This can happen in a spin-gapped system where the single-particle tunnelling is suppressed or for a sufficiently large inter-wire distance where both singleparticle and pair SC tunneling bare values are small and, therefore, can be neglected at not too low temperatures. In the latter case we have to consider, alongside with the (long-range) Coulomb interaction, the CDW perturbation only (the spin degrees of freedom simply double number of 'channels', $N \rightarrow 2 N$ ). It is reasonable to assume that the current-current inter-wire interaction is much weaker than the density-density one so that we put $\alpha_{\varphi}=0$ in Eqs. (24) and (28). We then find $\Delta^{\text {cdw }}$, Eq. (29), numerically and present our results for the $2 \mathrm{D}$ array in Fig. 1. The strength of the inter-wire interaction is bounded, $\left|\alpha_{\theta}\right| \leq \alpha_{\mathrm{WB}} \equiv(2 \ln (1+\beta))^{-1}$, because above the critical value $\alpha_{\mathrm{WB}}$ the Wentzel-Bardeen [37] instability occurs and this regime is beyond the applicability of our theory. Within the bounds, one immediately notices a competition between intraand inter-wire interactions. The graphs in Fig. 1a,b also illustrate that turning on the inter-wire interactions stabilises the SLL phase even for $K<1$. Thus, the SLL phase can be in principle observed in the multi-channel array provided that the inter-wire tunnelling necessary for the SP and SC perturbations is suppressed.

\section{Conclusions}

After analysing all allowed perturbations (single-particle tunneling and two-particle charge-density wave and Josephson couplings) in a SLL consisting of identical channels/wires with no magnetic field applied, we have shown that no physically reasonable inter-wire interaction can stabilize the model, i.e. support 'confinement' of particles and their pairs and the 'sliding' phase at the same moment. The situation is qualitatively similar in 2D- and 3D-packed arrays. The only possibility for such a description to be valid at the lowest temperature is the suppression of inter-wire hybridisation that can be achieved when either system is spin-gapped (attraction in the spin sector) or magnetic field is applied. 


\section{Acknowledgments}

VF and IVY acknowledge the hospitality of the Center for Theoretical Physics of Complex Systems, Daejeon, South Korea, where the main part of this project was performed. IVY's research was funded by the Leverhulme Trust Research Project Grant RPG-2016-044.

\section{References}

[1] Tomonaga S 1950 Prog. Theor. Phys. 5544

[2] Luttinger J M 1963 J. Math. Phys. 41154

[3] Haldane F D M 1981 J. Phys. C 14 2585; 1981 Phys. Rev. Lett. 471840

[4] Giamarchi T and Schulz H J 1988 Phys. Rev. B37 325

[5] Kane C L and Fisher M P A 1992 Phys. Rev. Lett.68 1220; 1992 Phys. Rev. B46 15233

[6] Furusaki A and Nagaosa N 1993 Phys. Rev. B47 4631

[7] Bockrath M et al 2001 Science 291283

[8] Ishii $\mathrm{H}$ et al 2003 Nature 426540

[9] Choi W B, Bae E, Kang D, Chae S, Cheong B, Ko J, Lee E and Park W 2004 Nanotechnology15 S512

[10] Mason N, Biercuk M J and Marcus C M 2004 Science 303655

[11] Melosh N A, Boukai A, Diana F, Gerardot B, Badolato A, Petroff P M and Heath J R 2003 Science300 112

[12] Slot E, Holst M A, van der Zant H S J and Zaitsev-Zotov S V 2004 Phys. Rev. Lett. 93176602

[13] Seeman N 2003 Nature 421427

[14] Zheng J, Birktoft J J, Chen Y, Wang T, Sha R, Constantinou P E, Ginell S L, Mao C and Seeman N C 2009 Nature 46174

[15] Neupert T, Chamon C, Mudry C and Thomale R 2014 Phys. Rev. B 90205101.

[16] Bockrath M et al 1999 Nature 397 598; Yao Z, Postma H W C, Balents L, and Dekker C 1999 Nature 402 273; Bachtold A et al 2001 Phys. Rev. Lett. 87 166801; Kanda et al 2004 Phys. Rev. Lett. 92 036801; Kar S et al 2009 ACS Nano 3207

[17] Zaitsev-Zotov S V et al 2000 J. Phys.: Condens. Matter 12 L303

[18] Venkataraman L, Hong Y S, and Kim P 2006 Phys. Rev. Lett. 96076601

[19] Slot Eet al 2004 Phys. Rev. Lett. 93176602

[20] Chandni U et al 2011 ACS Nano 5 8398; U. Chandni U et al 2013 Adv. Mater. 252486

[21] Aleshin et al 2004 Phys. Rev. Lett. 93196601

[22] Ning W eta l 2014 Appl. Phys. Lett. 105172603

[23] Wen X G 1990 Phys. Rev. B 426623

[24] Giamarchi T 2004 Quantum Physics in One Dimension (Clarendon Press, London)

[25] Golubović L and Golubović M 1998 Phys. Rev. Lett. 804341

[26] O'Hern C S, Lubensky T C and Toner J 1999 Phys. Rev. Lett. 832745

[27] Emery V, Fradkin E, Kivelson S and Lubensky T 2000 Phys. Rev. Lett. 852160

[28] Mukhopadhyay R, Kane C L and Lubensky T C 2001 Phys. Rev.B 63081103

[29] Kane C L, Mukhopadhyay R. and Lubensky T C 2002 Phys. Rev.Lett. 88036401

[30] Mukhopadhyay R, Kane C L, and Lubensky T C 2001 Phys. Rev. B 64045120

[31] Vishwanath A and Carpentier 2001 Phys. Rev.Lett. 86676

[32] Luther A and Emery V J 1974 Phys. Rev. Lett. 33589

[33] Seidel A and Lee D H 2005 Phys. Rev. B 71045113

[34] Yurkevich I V, Galda A, Yevtushenko O M and Lerner I V 2013 Phys. Rev. Lett. 110136405

[35] Yurkevich I V 2013 EPL 104 37004; 2017 Sci. Rep. 73550

[36] Kagalovsky V, Lerner IV and Yurkevich I V 2017 Phys. Rev.B 95205122 
[37] Wentzel G 1951 Phys. Rev. 83 168; Bardeen J 1951 Rev. Mod. Phys. 23261 